\title{
Clinical, Neuroimaging, and Genetic Features of the Patients with L-2-Hydroxyglutaric Aciduria
}

\author{
(1) Ebru Canda1, (1) Melis Köse1, (ㄱ) Havva Yazıcı1, (1) Esra Er1, (1) Cenk Eraslan2, (1) Sema Kalkan Uçar1, \\ ๑ Sara Habif3, ๑ Emin Karaca4, ๑ Hüseyin Onay4, ๑ Ferda Özkınay4, ๑ Mahmut Çoker ${ }^{4}$ \\ ${ }^{1}$ Ege University Faculty of Medicine, Department of Pediatrics, Division of Pediatric Metabolism and Nutrition, Izmir, Turkey \\ 2Ege University Faculty of Medicine, Department of Radiology, Izmir, Turkey \\ ${ }^{3}$ Ege University Faculty of Medicine, Department of Biochemistry, Izmir, Turkey \\ ${ }^{4}$ Ege University Faculty of Medicine, Department of Genetics, Izmir, Turkey
}

\begin{abstract}
Aim: L-2-hydroxyglutaric aciduria (L2HGA) is a rare autosomal recessive encephalopathy caused by mutations in the L-2-hydroxyglutarate dehydrogenase gene.

Materials and Methods: Here we discuss the clinical and molecular characteristics in patients with L2HGA.

Results: There were eight patients with L2HGA. Their median age was 16 (9.5-37) years. Five of them were female and three of them were male. The main symptoms of the patients were psychomotor retardation (8/8), cerebellar ataxia (5/8), extrapyramidal symptoms (7/8) and seizures (4/8). All patients had behavioral problems. Elevated urinary $\mathrm{L}-2$-hydroxy (L-2-OH) glutaric acid was detected and the median level of urine $\mathrm{L}-2-\mathrm{OH}$ glutaric acid at diagnosis was $146(60-1460 \mathrm{nmol} / \mathrm{mol}$ creat). Characteristic magnetic resonance imaging findings including subcortical cerebral white matter abnormalities with $\mathrm{T} 2$ hyperintensities of the dentate nucleus, globus pallidus and putamen were detected. Two patients had homozygous R335X, two patients had homozygous R2820, two patients had homozygous R302L and one patient had compound heterozygous P302L/A64T mutation in L-2-hydroxyglutarate dehydrogenase (L2HGDH) gene.

Conclusion: Because of the slow progression of the disease, the diagnosis of the patients is usually belated. L2HGA must be considered in the differential diagnosis based on clinical findings and specific findings in cranial magnetic resonance imaging. In our study, one of our patients has a novel mutation.

Keywords: L-2-hydroxyglutaric aciduria, ataxia, epilepsia, psychomotor retardation
\end{abstract}

\section{Introduction}

L-2-Hydroxyglutaric aciduria (L2HGA) is a very rare inherited metabolic disease with autosomal recessive inheritance [Online Mendelian Inheritance in Man (OMIM) \#236792]. Since its first description in 1980 by Duran et al., (1) many additional cases of various ethnical backgrounds have been reported. Affected patients have slowly progressive deterioration with cerebellar ataxia, mild or moderate mental retardation, and extrapyramidal and pyramidal symptoms, and seizures and variable macrocephaly (2). L2HGA is characterized by elevated levels of L-2- hydroxyglutaric acid ( $22 \mathrm{HG}$ ) in urine, cerebrospinal fluid (CSF), and, to a lesser extent, plasma. Increased levels of $L 2 H G$ are pathognomic for L2HGA $(1,3)$. Neuroimaging findings generally show subcortical leukoencephalopathy, cerebellar atrophy and changes in dentate nuclei and putamen (4). L-2-hydroxyglutarate (Figure 1) in nature suggests that it is endogenously produced in humans. This result is due to the findings that the excretion of L-2-hydroxyglutarate in patients with L2HGA is little affected by the diet and that L-2-hydroxyglutarate accumulates in cultured cells deficient in L-2-hydroxyglutarate dehydrogenase ( $\mathrm{L} 2 \mathrm{HGDH}$ (5). $\mathrm{L} 2 \mathrm{HGDH}$ belongs to a large family of flavin adenine dinucleotide (FAD)- 


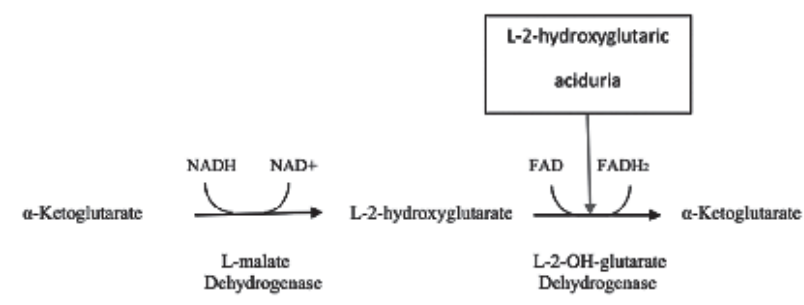

Figure 1. Oxidation of L-2-hydroxyglutaric acid to 2-ketoglutaric acid FAD: Flavin adenine dinucleotide, L-2-OH: L-2-hydroxy, NADH: Nicotinamide adenine dinucleotide

linked dehydrogenases and oxidases. L-2-hydroxyglutarate is formed from alpha-ketoglutarate (metabolite in the tricarboxylic acid cycle) by the side activity of the mitochondrial L-malate dehydrogenase. L-2-hydroxyglutarate accumulation is toxic to the human brain, causing a leukoencephalopathy and increasing the sensibility to develop tumours (6). Episodes of acute metabolic decompensation do not occur, and brain damage is not related to acidosis in L2HGA patients. It's a difference from other forms of organic acidurias (7). The disease-causing gene, $\angle 2 H G D H$ gene $(\angle 2 H G D H)$, and its first pathogenic mutations were identified in 2004 (8,9). Treatment of $\mathrm{L} 2 \mathrm{HGA}$ is under investigation; case reports have described positive effects of FAD sodium in combination with levocarnitine chloride in one patient (10) and riboflavin, a precursor of FAD, in another patient (11). The present study describes the clinical presentation and mutation analysis and follow up findings of our patients with L2HGA at Ege University Pediatric Metabolism and Nutrition Unit.

\section{Materials and Methods}

Eight patients who were diagnosed with L2HGA between the years of 2004-2016 were included in this study. Patients' demographical features; age, sex and age at diagnosis, consanguinity; clinical findings (such as; psychomotor retardation, loss of skills, extrapyramidal symptoms, seizures, behavioural problems) and head circumference (the presence of macrocephaly) were recorded. Urine 2-hydroxy (2-OH) glutaric acid levels at the diagnosis and L2HGDH gene analysis were analysed retrospectively. Radiological features were retrospectively included to the analysis.

\section{Results}

Median age of the patients was 17 (9.5-37) years and median age at diagnosis was 8 years (2-25 years). Female/male ratio of the patients was $5 / 3$. Patient 1,2 and Patient 6,7 were sibling. The main symptoms of the patients were psychomotor retardation (8/8), cerebellar ataxia (5/8), extrapyramidal symptoms (7/8), and seizures (4/8). All patients have behavioural problems. Five parents had consanguinity marriage. Five patients' head circumferences were known and none of them had macrocephaly. Clinical and demographical findings of the patients were detailed in Table I. Patient 6 diagnosed at the age of 25 years. Speech difficulties developed at the earlier ages, walking difficulties and seizures developed at the age of 25 years. Her sister with the same diagnosis (follow up at different clinic) had speech delay at the early childhood and learning difficulties at school age and tremor at the age of 15 years. All patients could walk without support before they lost of walking ability. Three patients' unsupported walking ages were not applicable. Patient 3 had psychomotor retardation and behavioural problems. He had milder clinic symptoms. Elevated urinary $2-\mathrm{OH}$ glutaric acid was detected and median level of the urine 2-OH glutaric acid at the diagnosis was 146 (60-1460 $\mathrm{nmol} / \mathrm{mol}$ creat). Two patients have homozygous R335X, two homozygous R282Q, two homozygous R302L, and one compound heterozygous P302L/A64T mutation in $\mathrm{L} 2 \mathrm{HGDH}$ gene. One of the patient's $\angle 2 H G D H$ gene analysis was not applicable. Detail diagnostic laboratory findings of the patients were given in Table II. Characteristic magnetic resonance imaging (MRI) findings including subcortical cerebral white matter (WM) abnormalities with T2 hyperintensities of the dentate nucleus, globus pallidus, putamen was detected. WM hyperintensities were detected in all patients. Dentate nucleus hyperintensities were detected in 3 patients, basal ganglion hyperintensities were detected in 5 patients and cystic encephalomalasia in one patient. Cranial MRI findings of the patient were detailed in Table III. In patient 2 cranial MRI shows WM involvement, cerebral atrophy and spongiform changes (Figure 2). In patient 3, cranial MRI reveals bilateral globus pallidus and caudate nucleus involvements (Figure 3 ).

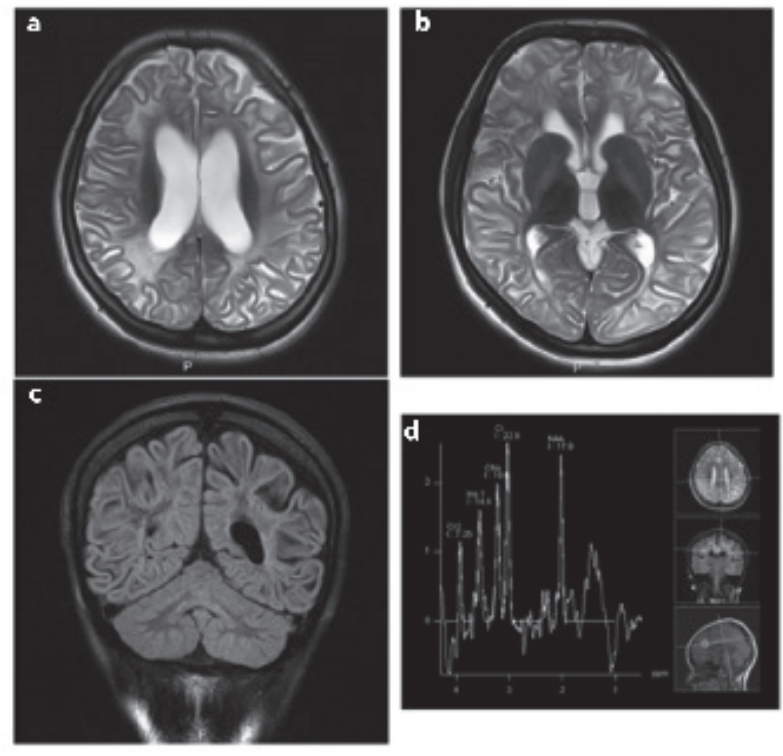

Figure 2. Axial T2 $(a, b)$, coronal flair images, c) showing diffuse white matter hyperintentisities. Cerebral cortex and basal ganglions are spared. Multivoxel magnetic resonance spectroscopy images, d) showing reduced $\mathrm{N}$-acetyl-aspartate level at the level of affected frontal white matter 


\section{Discussion}

L2HGA is a very rare inherited metabolic disease with autosomal recessive inheritance (OMIM \#236792). Affected individuals only have neurological manifestations, including mild to moderate psychomotor retardation, cerebellar ataxia, variable macrocephaly, and epilepsy $(7,12-14)$. In our study similar to the literature the main symptoms of the patients were psychomotor retardation, cerebellar ataxia, extrapyramidal symptoms, and seizures. In the literature large studies demonstrated that during the disease course the main clinical findings were developmental delay (93\%),
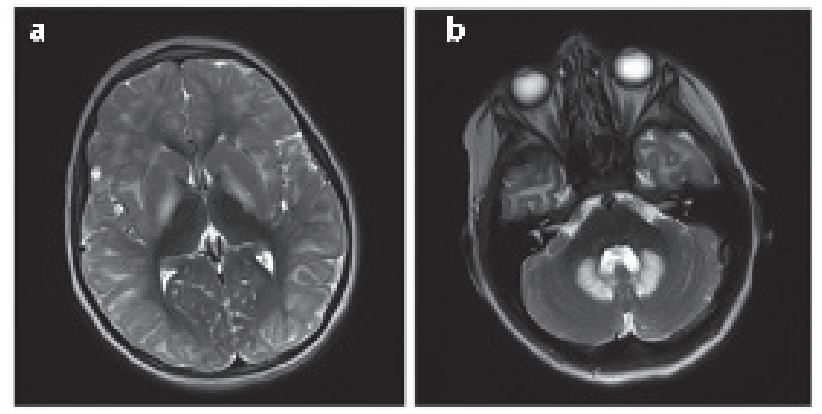

Figure 3. Axial T2 images showing hyperintentisities; a) bilateral globus pallidus, b) bilateral dentate nucleus cerebellar ataxia (82\%), epilepsy (72\%), and macrocephaly $(48 \%)$ (2). It is interesting that in our study the available head circumferences were all normal. We couldn't get an information about the head circumferences of the 3 patients. In the earlier stages of the disease hypotonia was most prevalent and spasticity in the latter stages of disease in their cohort. Neurological decompensation (e.g., loss of skills and the development of speech deficits) was also present in a quarter of the patients (15). In our study loss of the skills were detected in 5 patients and all of them developed speech deficits. Also, all patients had behavioural problems. The clinical symptoms usually recognise during infancy or childhood. The cases in the literature reported as adult onset were diagnosed as adult onset but, in retrospect, the patients had symptoms during childhood (2). Patient 6 diagnosed at the age of 25 years. Speech difficulties developed at the earlier ages, walking difficulties and seizures developed at the age of 25 years. Her sister with the same diagnosis had speech delay at the early childhood and learning difficulties at school age and tremor at the age of 15 years. Mild symptoms may explain the late diagnosis ages. Seizures manifest in most of the patients (7). In our study 4 of the patients had the history of seizures. Patient 1 had the history of febrile seizures between the age of 3-4 years.

Table I. Demographical and clinical findings of the patients with L-2-hydroxyglutaric aciduria

\begin{tabular}{|c|c|c|c|c|c|c|c|c|}
\hline Patient no & $1^{\text {a }}$ & $2^{\mathrm{a}}$ & 3 & 4 & 5 & $6^{b}$ & $7^{\mathrm{b}}$ & 8 \\
\hline Age (year) & 19 & 11 & 9.5 & 14 & 32 & 37 & 25 & 13 \\
\hline Age at diagnosis (year) & 6 & 6 & 7 & 2 & 16 & 25 & 12 & 9 \\
\hline Gender & Female & Female & Male & Female & Female & Male & Female & Male \\
\hline Consanguinity & + & + & + & - & + & + & + & + \\
\hline Head circumferences & $\begin{array}{l}55.5 \mathrm{~cm} \\
(50-98 \mathrm{p})\end{array}$ & $\begin{array}{l}52.5 \mathrm{~cm} \\
(50-98 \mathrm{p})\end{array}$ & $\begin{array}{l}52 \mathrm{~cm} \\
(50-98 \mathrm{p})\end{array}$ & $\begin{array}{l}55 \mathrm{~cm} \\
(50-98 p)\end{array}$ & NA & NA & NA & $\begin{array}{l}55 \mathrm{~cm} \\
(50-98 \mathrm{p})\end{array}$ \\
\hline Psychomotor retardation & - & - & + & + & + & + & + & + \\
\hline $\begin{array}{l}\text { Unsupported walking age } \\
\text { (months) }\end{array}$ & 13 & 17 & 24 & 36 & NA & NA & 18 & 12 \\
\hline Loss of skills & + & + & - & + & - & + & + & - \\
\hline Behavioural problems & + & + & + & + & + & + & + & + \\
\hline Seizures & + & - & - & - & - & + & + & + \\
\hline Cerebellar ataxia & + & + & - & + & + & + & - & - \\
\hline Extrapyramidal symptoms & + & + & - & + & + & + & + & + \\
\hline
\end{tabular}

Table II. Diagnostic laboratory findings of the patients with L-2-hydroxyglutaric aciduria

\begin{tabular}{|l|l|l|l|l|l|l|l|l|}
\hline Patient no & 1 & 2 & 3 & 4 & 5 & 6 & 7 & 8 \\
\hline $\begin{array}{l}\text { Urine 2-OH glutaric } \\
\text { acid (mmol/mol/ } \\
\text { creat) }\end{array}$ & 150 & 250 & 308 & 143 & 78 & 76 & 1460 & 135 \\
\hline $\begin{array}{l}\text { L2HGDH gene } \\
\text { mutations }\end{array}$ & $\begin{array}{l}\text { R335X (CGA>TGA) } \\
\text { homozygous }\end{array}$ & $\begin{array}{l}\text { R335X (CGA>TGA) } \\
\text { homozygous }\end{array}$ & $\begin{array}{l}\text { p.P302L (c905C>T) } \\
\text { /p.A64T (c.1906G>A) } \\
\text { compound heterozygote }\end{array}$ & $\begin{array}{l}\text { p.P302L } \\
\text { (c905 C>T) } \\
\text { homozygous }\end{array}$ & $\begin{array}{l}\text { R2820 } \\
\text { c.1003C>T } \\
\text { homozygous }\end{array}$ & $\begin{array}{l}\text { R2820 } \\
\text { homozygous }\end{array}$ & $\begin{array}{l}\text { NA } \\
\text { (c905 C>T) } \\
\text { homozygous }\end{array}$ \\
\hline
\end{tabular}

NA: Not applicable, L2HGDH: L-2-hydroxyglutarate dehydrogenase, 2-OH: 2-hydroxy 


\begin{tabular}{|c|c|}
\hline Patient no & Cranial MRI findings \\
\hline 1 & $\begin{array}{l}\text { Bilateral cerebral atrophy, white matter T2 hyperintensities } \\
\text { MR-S decreased NAA and choline peak }\end{array}$ \\
\hline \multirow[t]{4}{*}{2} & Bilateral cerebral atrophy, white matter T2 hyperintensities \\
\hline & $\begin{array}{l}\text { Bilateral dentate nucleus and capsula externa diffusion } \\
\text { restriction }\end{array}$ \\
\hline & Substantia nigra T2 hyperintensities \\
\hline & MR-S decreased NAA and choline peak \\
\hline \multirow[t]{2}{*}{3} & Bilateral subcortical white matter hyperintensities \\
\hline & MR-S decreased NAA and choline peak \\
\hline \multirow[t]{3}{*}{4} & $\begin{array}{l}\text { Bilateral cerebral white matter T2 hyperintensities and } \\
\text { diffusion restriction }\end{array}$ \\
\hline & $\begin{array}{l}\text { Bilateral putamen, bilateral caudate nucleus T2 } \\
\text { hyperintensities and diffusion restriction }\end{array}$ \\
\hline & Bilateral internal and external capsula hyperintensities \\
\hline 5 & Bilateral cerebral white matter T2 hyperintensities \\
\hline 6 & $\begin{array}{l}\text { Bilateral cerebral hemisphere at basal ganglion levels } \\
\text { cystic encephalomalasia }\end{array}$ \\
\hline 7 & $\begin{array}{l}\text { Bilateral subcortical white matter T2 hyperintensities } \\
\text { Dentate nucleus T2 hyperintensities }\end{array}$ \\
\hline \multirow[t]{3}{*}{8} & Bilateral white matter T2 hyperintensities \\
\hline & Basal ganglion T2 hyperintensities \\
\hline & Bilateral nucleus dentate hyperintensities \\
\hline
\end{tabular}

MR-S: $\mathrm{N}$-acetyl aspartate, MRI: Magnetic resonance imaging, NAA: N-acetyl-aspartate

The diagnosis is supported by increased levels of L-2hydroxyglutarate acid in urine, plasma, and cerebro-spinal fluid (1). Some patients show increased levels of L-lysine and pipecolate in CSF suggesting a defect in alternative degradation pathway of lysine (16). We had not performed CSF analysis in our patients and plasma lysine levels were normal. We detected high 2-OH glutaric acid levels in all patients. Determination of $\mathrm{L}-2-\mathrm{OH}$ isoform could not have performed. The diagnosis of the disease confirmed by $\mathrm{L} 2 \mathrm{HGDH}$ gene analysis in 6 patients. $\mathrm{L} 2 \mathrm{HGDH}$ gene analysis of the patient 7 was not applicable but she has high urine 2-OH glutaric acid levels and also she has the sibling with genetically diagnosed L2HGA (patient 6). Cranial MRI findings generally show subcortical leukoencephalopathy, cerebellar atrophy and changes in basal ganglion, in dentate nucleus (17). Steenweg et al. (17) investigated 56 patients with L2HGA and they found that with increasing disease duration, WM abnormalities and basal ganglia signal intensity abnormalities become more diffuse and cerebral WM atrophy arises. The few descriptions of histologic brain findings in L2HGA reported WM spongiosis, demyelination, and cystic degeneration, mostly in the subcortical regions (17). In our study all patients had WM changes on cranial MR. Three patients had bilateral dentate nucleus hyperintensities. Cranial MR investigation of patient 6 revealed cystic encephalomalasia. Işıkay (18) reported the case with L2HGA who had macrocephaly and cerebral multicystic lesions. Few data on proton MR spectroscopic (MR-S) changes are available. Anghileri et al. (19) detected 2 hydroxy glutarate peak in the WM of the three L2HGA patients. In our study decreased $\mathrm{N}$-acetyl aspartate and choline peaks were detected on MR-S. Successful therapeutic trials have been reported in patients L2HGA. The patient described by Yilmaz (11) a 16 years old boy, had been treated with riboflavin for nearly 2 years and during treatment at $100 \mathrm{mg} /$ day partial improvement in his cognitive and motor performances was observed and urinary secretion was decreased. Samuraki et al. (10) described 43 years old woman with L2HGA who was treated with FAD (30 mg/day) and carnitine. Significant improvement in her tremor and dystonia was detected and decreased urinary excretion of L2 hydroxy glutarate was detected (10). In our study half of the patients treated with riboflavin and due to the irregular follow up its difficult to give a comment on the treatment response. But we observed clinical stability. Two of our patients (siblings) have homozygous R335X (nonsense mutation) and 2 patients had homozygous p.P302L mutations. These mutations were reported in Turkish patients by Topcu et al. (8). Two patients had homozygous R2820 mutations which was previously reported by Samuraki et al. (10) Patient 3 had the p.P302L (c905 C>T)/p.A64T (c.1906G>A) compound heterozygote mutation. According to our knowledge p.A64T (c.1906G>A) had not been reported. Most patients reach adulthood. Increased incidence of brain tumours has been reported (20-22). A major difference between L2HGA and other dicarboxylic acidurias is that it is associated with a significant increase in the incidence of brain tumours. Six cases who developed brain tumour have been reported for a total of 100 known patients (6).

\section{Conclusion}

The symptoms of L2HGA are usually progressive. Some patients with milder clinical findings start having problems late in life. Early diagnosis of the patients may be useful because riboflavin treatment increases the activity of proteins in some patients. Regular follow up is also important because of the increased incidence of brain tumours.

\section{Ethics}

Informed Consent: Consent form was filled out by all participants.

Peer-review: External and internal peer-reviewed.

\section{Authorship Contributions}

Surgical and Medical Practices: E.C., M.K., H.Y., E.E. Concept: E.C., M.Ç., S.K.U., Design: E.C., M.Ç., Data Collection or Processing: E.C., S.K.U., C.E., S.H., Analysis or Interpretation: E.K., H.O., F.̈., Literature Search: E.C., S.K.U., Writing: E.C., M.C.., S.K.U.

Conflict of Interest: No conflict of interest was declared by the authors. 
Financial Disclosure: The authors declared that this study received no financial support.

\section{References}

1. Duran M, Kamerling JP, Bakker HD, van Gennip $A H$, Wadman SK. L-2-Hydroxyglutaric aciduria: an inborn error of metabolism? J Inherit Metab Dis 1980;3:109-12.

2. Steenweg ME, Jakobs C, Errami A, et al. An overview of L-2hydroxyglutarate dehydrogenase gene (L2HGDH) variants: a genotype-phenotype study. Hum Mutat 2010;31:380-90.

3. Barth PG, Hoffmann GF, Jaeken JJ, et al. L-2-Hydroxyglutaric aciduria: a novel inherited neurometabolic disease. Ann Neurol 1992;32:66-71.

4. Steenweg ME, Salomons GS, Yapici Z, et al. L-2Hydroxyglutaric aciduria: pattern of MR imaging abnormalities in 56 patients 1. Radiology 2009;251;856-65.

5. Struys EA, Verhoeven NM, Roos B, Jakobs C. Diseaserelated metabolites in culture medium of fibroblasts from patients with D-2-hydroxyglutaric aciduria, L-2-hydroxyglutaric aciduria, and combined D/L-2-hydroxyglutaric aciduria. Clin Chem 2003;49:1133-8.

6. Van Schaftingen E, Rzem R, Veiga-da-Cunha M. L:-2Hydroxyglutaric aciduria, a disorder of metabolite repair. $J$ Inherit Metab Dis 2009;32:135-42.

7. Topçu M, Aydin OF, Yalcinkaya C, et al. L-2-Hydroxyglutaric aciduria: a report of 29 patients. Turk J Pediatr 2005;47:1-7.

8. Topcu M, Jobard F, Halliez S, et al. L-2-Hydroxyglutaric aciduria: identification of a mutant gene C14orf160, localized on chromosome 14q22.1. Hum Mol Genet 2004;13:280311.

9. Rzem R, Veiga-da-Cunha M, et al. A gene encoding a putative FAD-dependent L-2-hydroxyglutarate dehydrogenase is mutated in L-2-hydroxyglutaric aciduria. Proc Natl Acad Sci U S A 2004;101:16849-54.

10. Samuraki $M$, Komai $K$, Hasegawa $Y$, et al. A successfully treated adult patient with L-2-hydroxyglutaric aciduria. Neurology 2008;70:1051-2.
11. Yilmaz K. Riboflavin treatment in a case with I-2-hydroxyglutaric aciduria. Eur J Paediatr Neurol 2009;13:57-60.

12. Barbot C, Fineza I, Diogo L, et al. L-2-Hydroxyglutaric aciduria: clinical, biochemical and magnetic resonance imaging in six Portuguese pediatric patients. Brain Dev 1997;19:268-73.

13. Barth PG, Hoffmann GF, Jaeken J, et al. L-2-Hydroxyglutaric acidaemia: clinical and biochemical findings in 12 patients and preliminary report on L-2-hydroxyacid dehydrogenase. J Inherit Metab Dis 1993;16:753-61.

14. Moroni I, D'Incerti L, Farina L, Rimoldi M, Uziel G. Clinical, biochemical andneuroradiological findings in L-2hydroxyglutaric aciduria. Neurol Sci 2000;21:103-8.

15. Kranendijk M, Struys EA, Salomons GS, Van der Knaap MS, Jakobs C. Progress in understanding 2-hydroxyglutaric acidurias. J Inherit Metab Dis 2012;35:571-87.

16. de Klerk JB, Huijmans JG, Stroink H, et al. L-2-hydroxyglutaric aciduria: clinical heterogeneity versus biochemical homogeneity in a sibship. Neuropediatrics 1997;28:314-7.

17. Steenweg ME, Salomons GS, Yapici Z, et al. L-2Hydroxyglutaric aciduria: pattern of MR imaging abnormalities in 56 patients. Radiology 2009;251:856-65.

18. Işıkay S. Cerebral multicystic lesions in a child with L-2 hydroxyglutaric aciduria: a rare disease and a rare association. Pediatr Neurol 2014;50:197-8.

19. Anghileri E, Bertolino N, Salsano E, et al. In-vivo brain H1-MR-Spectroscopy identification and quantification of 2-hydroxyglutarate in L-2-Hydroxyglutaric aciduria. Brain Res 2016;1648:506-11.

20. Aghili 1, Zahedi F, Rafiee E. Hydroxyglutaric aciduria and malignant brain tumor: a case report and literature review. J Neurooncol 2009;91:233-6.

21. Haliloglu G, Jobard F, Oguz KK, et al. L-2-hydroxyglutaric aciduria and brain tumors in children with mutations in the L2HGDH gene: neuroimaging findings. Neuropediatrics 2008;39:119-22.

22. Moroni I, Bugiani M, D'Incerti L, et al. L-2-hydroxyglutaric aciduria and brain malignant tumors: a predisposing condition? Neurology 2004;62:1882-4. 